\title{
STRATIGRAPHER: MAKING AND USING LITHOLOGS IN $R$
}

Devleeschouwer X. 1, Da Silva A.-C. 2, Boulvain F. 2 \& Wouters S. 1, 2*

1. O.D. Earth and History of Life, Royal Belgian Institute of Natural Sciences

2. Sedimentary Petrology, University of Liège

* Corresponding and presenting author: sebastien.wouters@doct.uliege.be 
This presentation is an introduction to $R$ and to Stratigraphe $R$. It was originally supposed to be a poster only on StratigrapheR presented at EGU 2020. As the current situation of confinement made the physical EGU 2020 impossible, and brought the virtual EGU, more freedom was given to provide supplementary material.

We therefore present what was supposed to be a presentation available on the web, which is made to support general discussions [what we wanted to call a R'staurant] on R software development concepts for geology purposes.

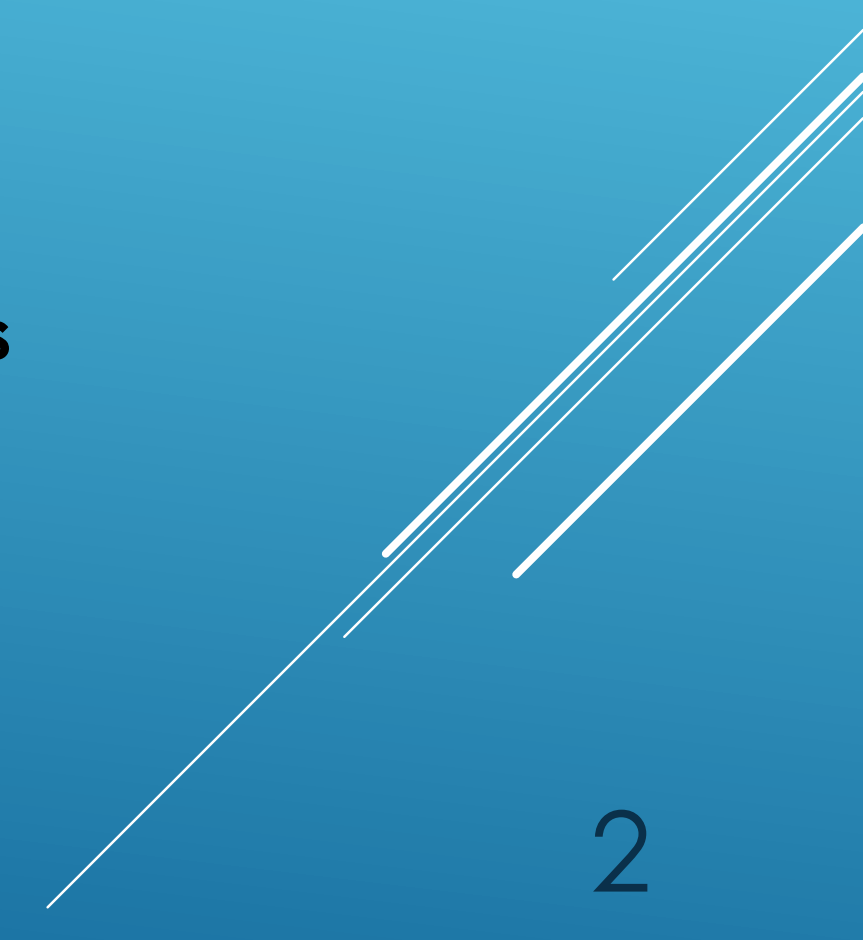




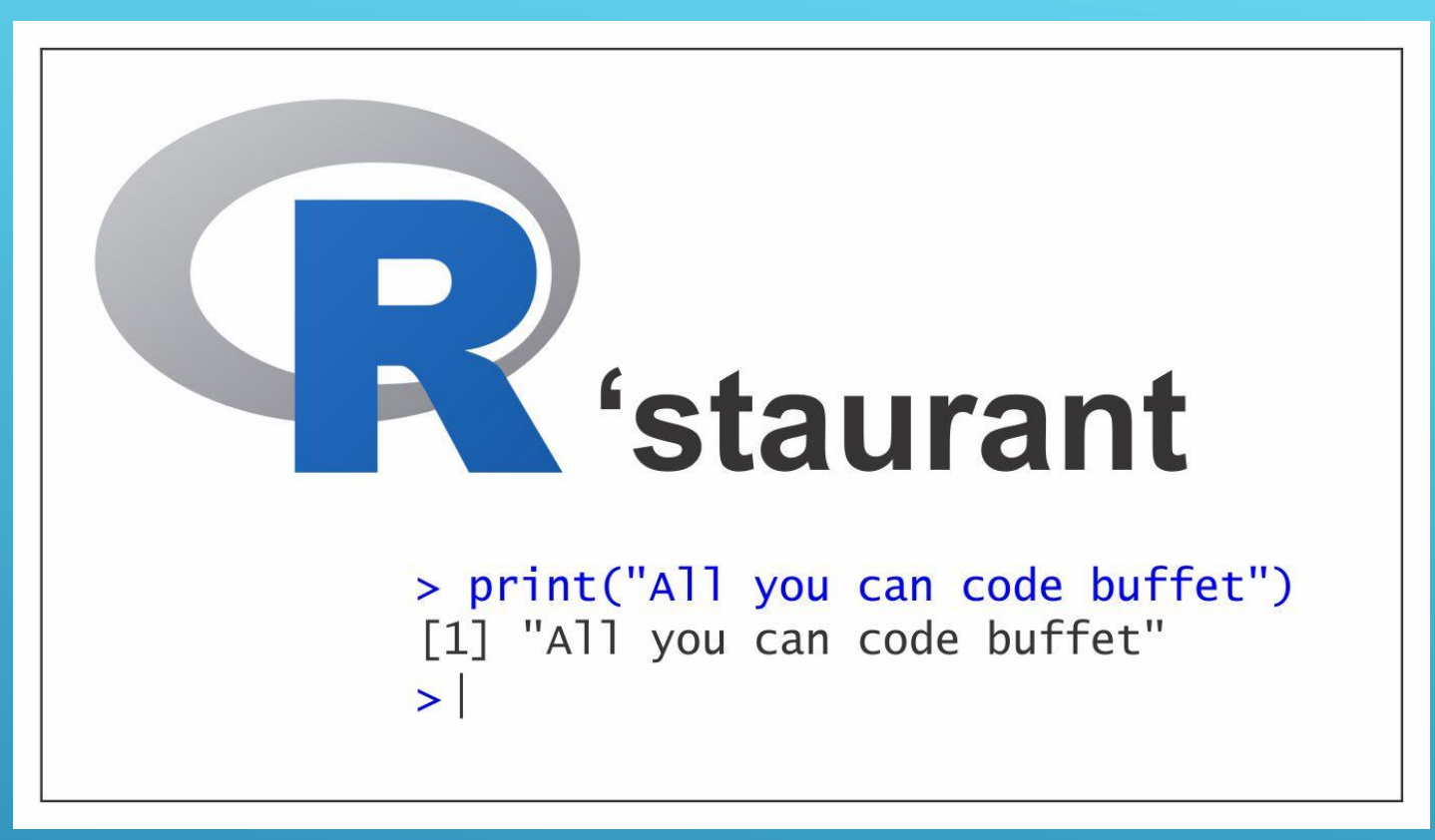

This is the R'staurant supportive power point, designed to provide a basis in $\mathbf{R}$ for geologists:

- Quick bullet points for fundamental R concepts

- Starting manual for StratigrapheR

- Links to other internet resources

- In development (v. 0.0.1 A, for EGU 2020)

- Open to any suggestion or correction 


\section{CHAPTERS}

- Introduction...............................5

• General R coding.............................11

- Basic tutorials.................................12

- Efficient R coding.............................13

• Avoiding common problems................17

• numeric values...................................18

• text and characters...........................20

• StratigrapheR.................................22

- PLEASE HELP ME, my code does not work and I am desperately stuck !t!.....41 


\title{
INTRODUCTION
}

\author{
Getting the necessary software
}




\section{WHY R ?}

It is free

It is awesome

It will change your life

- It can make software evolve by a community effort

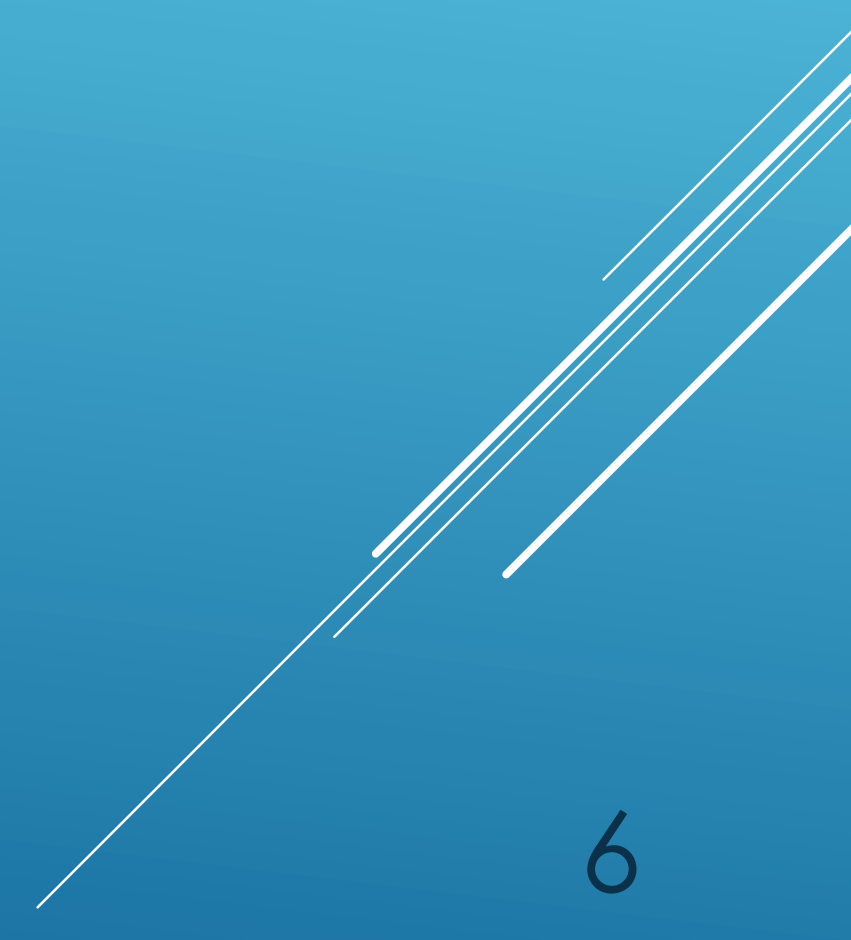




\section{HOW TO GET R AND RSTUDIO?}

- Download $\mathbf{R}$ on the Comprehensive R Archive Network (CRAN) https://cran.r-project.org/ (or type 'download $R$ ' in your browser)

- Download RStudio on the RStudio website https://rstudio.com/products/rstudio/\#rstudio-desktop (or type 'rstudio' in your browser)

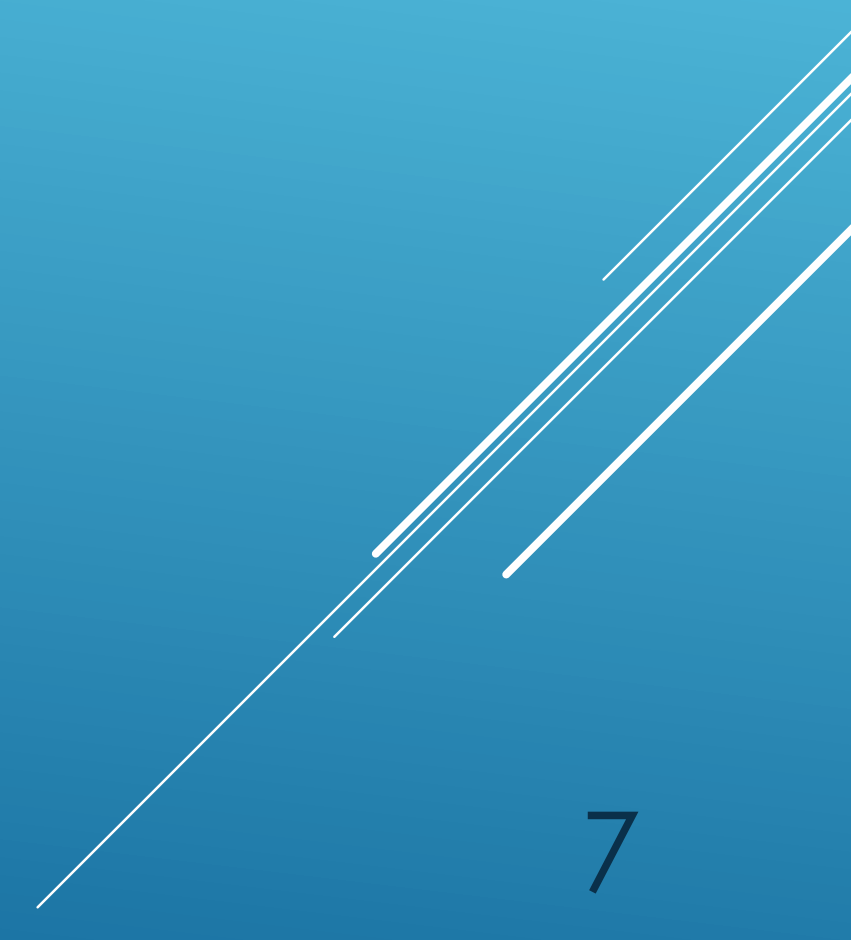


RStudio is a free scripting interface for $\mathrm{R}$

It is widely accepted as the norm for $\mathrm{R}$ interfacing

- It will make your life easier

\section{(8) RStudio}

File Edit Code View Plots Session Build Debug Profile Tools Help

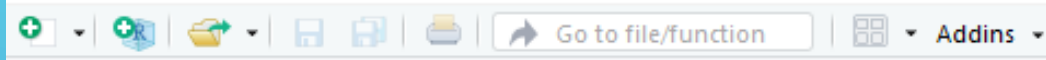

(2) Script $1 . R \times$

$\square \square$

1 \# code

a $<-1: 20$

4 b $<--a$

6 plot $\left(a, b\right.$, pch $=19$, type $={ }^{\circ}$ " $)$

6:33 (Top Level)

Console $\mathrm{C} /$ /Users/Seb/Desktop/Publications/3. R'staurant/

R version 3.6.1 (2019-07-05) -- "Action of the Toes"

Copyright (C) 2019 The R Foundation for statistical computing Platform: x86_64-w64-mingw32/x64 (64-bit)

$R$ is free software and comes with ABSOLUTELY NO WARRANTY.

You are welcome to redistribute it under certain conditions.

Type 'license()' or 'licer $\mathrm{C} 2 \mathrm{O}$ ' for distribution details.

$R$ is a collaborative project with many contributors.

Type 'contributors ()' for more information and

citation ()$^{\prime}$ on how to cite $R$ or $R$ packages in publications.

Type 'demo()' for some demos, 'help()' for on-line help, or

'help.start ()$^{\prime}$ for an HTML browser interface to help.

Type ' $q()^{\prime}$ ' to quit $R$.

$>$ \# Code

a $<-1: 20$

$a<-1: 20$
$b<--a$

plot $(a, b$, pch $=19$, type $=" o ")$

Evironment History Connections

R. Project: (None)

$\rightarrow$ 日 $\rightarrow$ Import Dataset v |

$=$

Global Environment

values

a

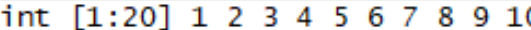

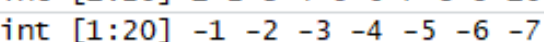

Files Plots Packages Help Viewer

$\square \square$

Zoom - IT Export

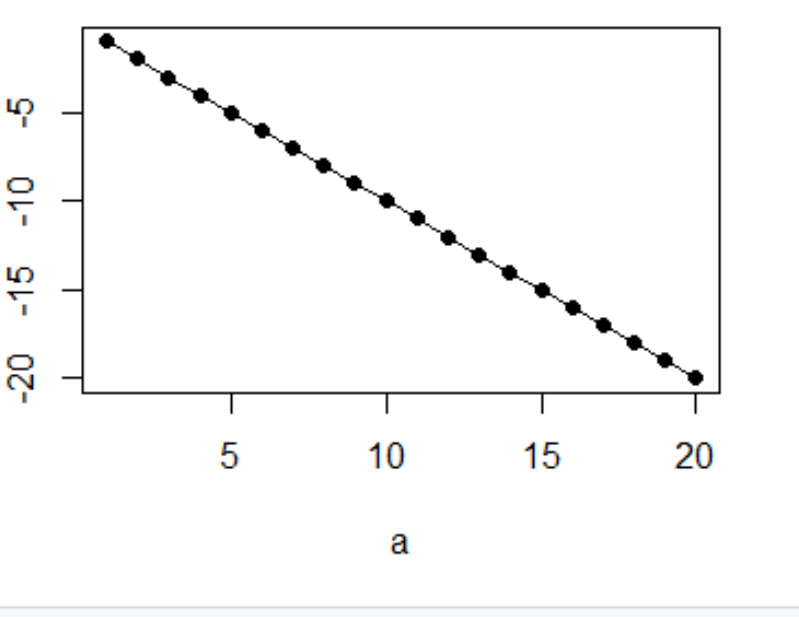




\section{HOW TO GEI NEW PACKAGES?}

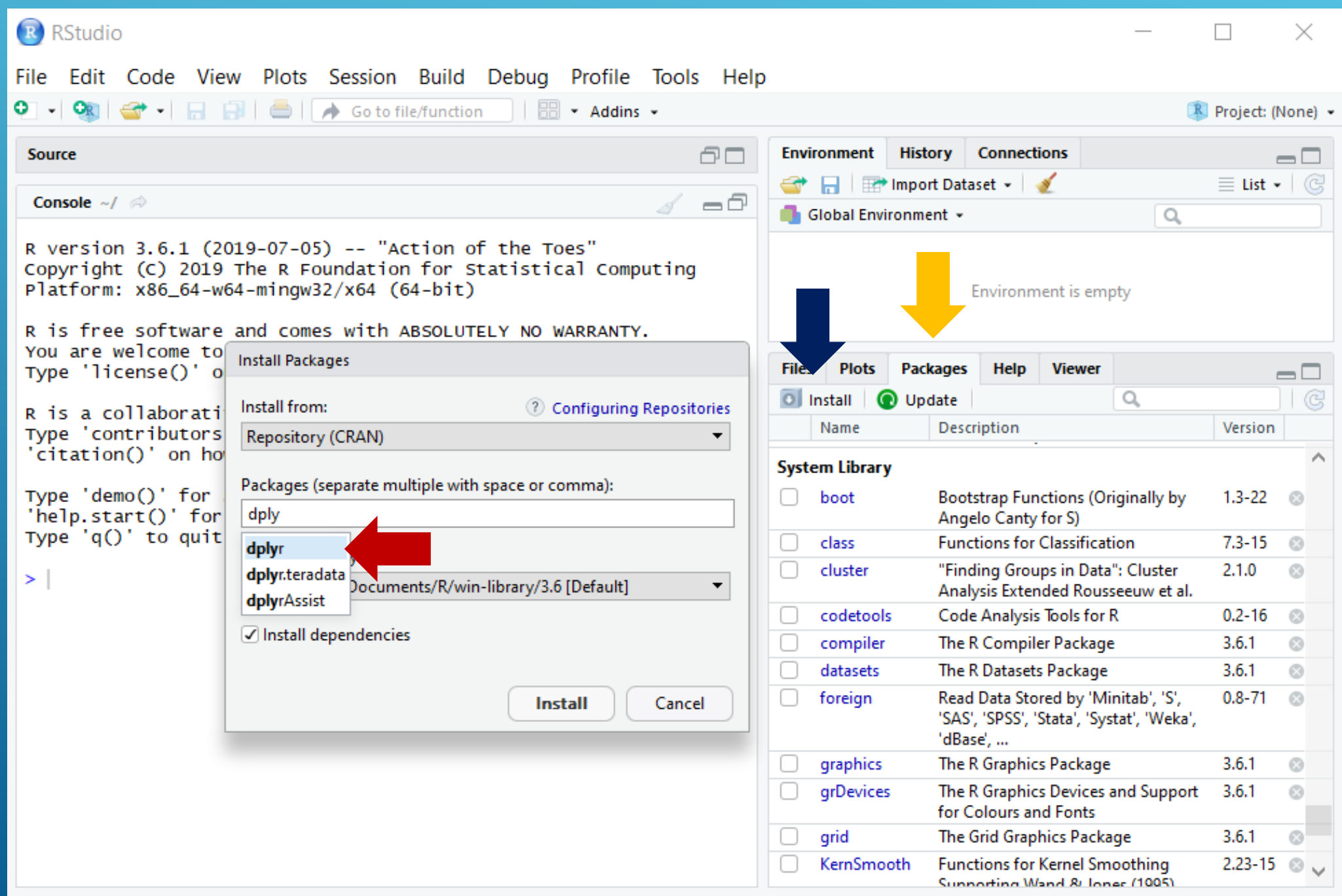

- Go to Packages

- Click on Install

- Type the name of the package you want to install

\begin{tabular}{|c|c|}
\hline $\begin{array}{l}\text { Bootstrap Functions (Originally by } \\
\text { Angelo Canty for S) }\end{array}$ & $8-2$ \\
\hline Functions for Classification & $7.3-15$ \\
\hline $\begin{array}{l}\text { "Finding Groups in Data": Cluster } \\
\text { Analysis Extended Rousseeuw et al. }\end{array}$ & 2.1 .0 \\
\hline Code Analysis Tools for $R$ & $0.2-16$ \\
\hline The R Compiler Package & 3.6 .1 \\
\hline The R Datasets Package & 3.6 .1 \\
\hline $\begin{array}{l}\text { Read Data Stored by 'Minitab', 'S', } \\
\text { 'SAS', 'SPSS', 'Stata', 'Systat', 'Weka', } \\
\text { 'dBase', ... }\end{array}$ & $0.8-71$ \\
\hline The R Graphics Package & 3.6 .1 \\
\hline $\begin{array}{l}\text { The R Graphics Devices and Support } \\
\text { for Colours and Fonts }\end{array}$ & 3.6.1 \\
\hline The Grid Graphics Package & 3.6 .1 \\
\hline $\begin{array}{l}\text { Functions for Kernel Smootl } \\
\text { Sunnnetinn Wand \& Inner } 1\end{array}$ & $2.23-15$ \\
\hline
\end{tabular}

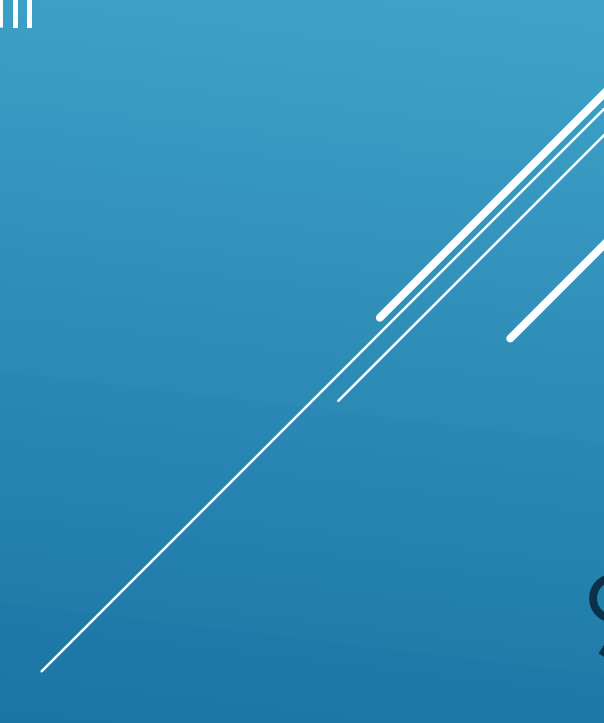




\section{HOW TO GET NEW PACKAGES?}

- To get packages you can also run the function install.packages(), with the name of the package:

install.packages ("StratigrapheR")

- To upload the package content and be able to work with it, you need to invoke it every time you open R:

library (StratigrapheR) 


\section{GENERAL R CODING}

A few general things to keep in mind

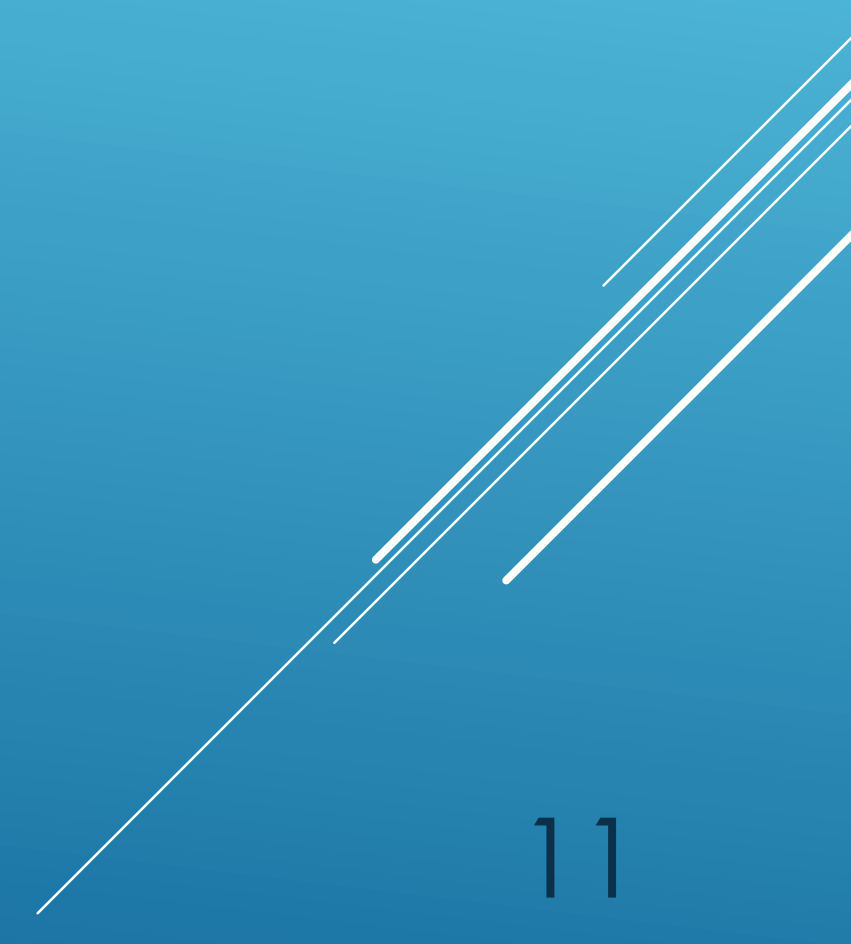


- Great R basic tutorials exist, for instance:

https://cyclismo.org/tutorial/R/

- You can usually find good tutorials by googling them, for instance I got the following by googling ' $R$ basic plots':

https://sites.harding.edu/fmccown/r/

\section{BASIC TUTORIALS}


- $\mathrm{R}$ is an interpreted language.

\section{What does that mean?}

- The code is not compiled: it runs directly

\section{How is that done?}

- The base R functions are pre-compiled, and any script ultimately redirects towards these pre-compiled functions

\section{Why should I care ?}

This affects the speed of computations

\section{EFFICIENT R CODING}


> How do I code R scripts to make computations fast?

The general rule is to avoid loops: 'for' loops and 'repeat' loops.

Such loops are efficient in compiled languages such as $C$ or

Fortran, however they take a lot of time in $\mathbf{R}$.

- How do I avoid loops?

Find basic functions in $\mathbf{R}$ that do what you want

- How can I find such functions?

Google can offer a good start, Stack overflow is also a good way to find answers to questions that were already asked by other users;

https://stackoverflow.com/questions/tagged/r

EFFICIENT R CODING

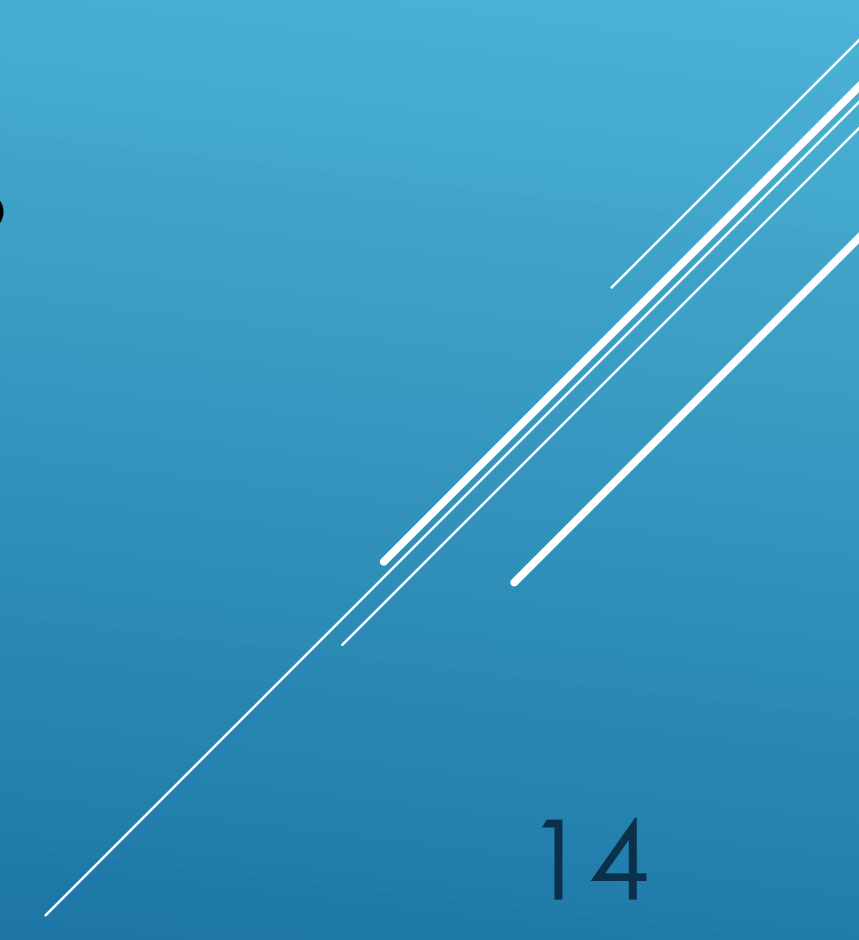


- Could you show me an example of a function that allows to avoid loops?

Sure thing, here is how to compute the cumulative sum of the sequence of 1 to 10:

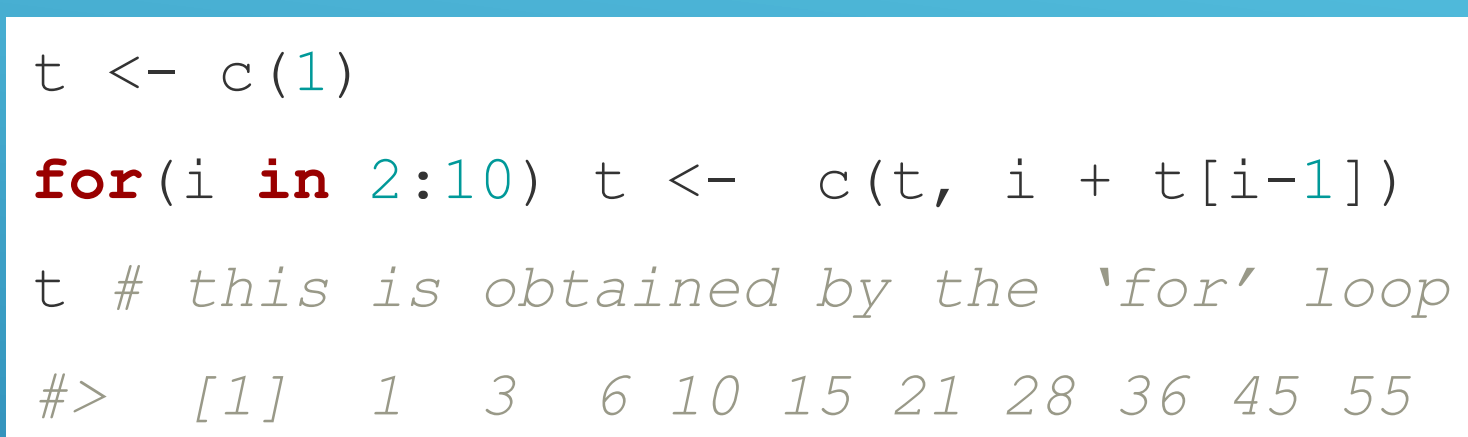

\section{EFFICIENT R CODING}


- What if I cannot avoid a loop?

Try to confine the looped part of the code to the strict minimum. You can further isolate the loops using the apply() family of functions, that loops functions more efficiently. For a tutorial on these functions:

https://www.guru99.com/r-apply-sapply-tapply.html

\section{EFFICIENT R CODING}




\section{AVOIDING COMMON PROBLEMS}

A few things I wish I knew before getting into $R$, and that allow you to avoid common problems that beginners will eventually run into, and that drive you INSANE trying to understand what is happening!

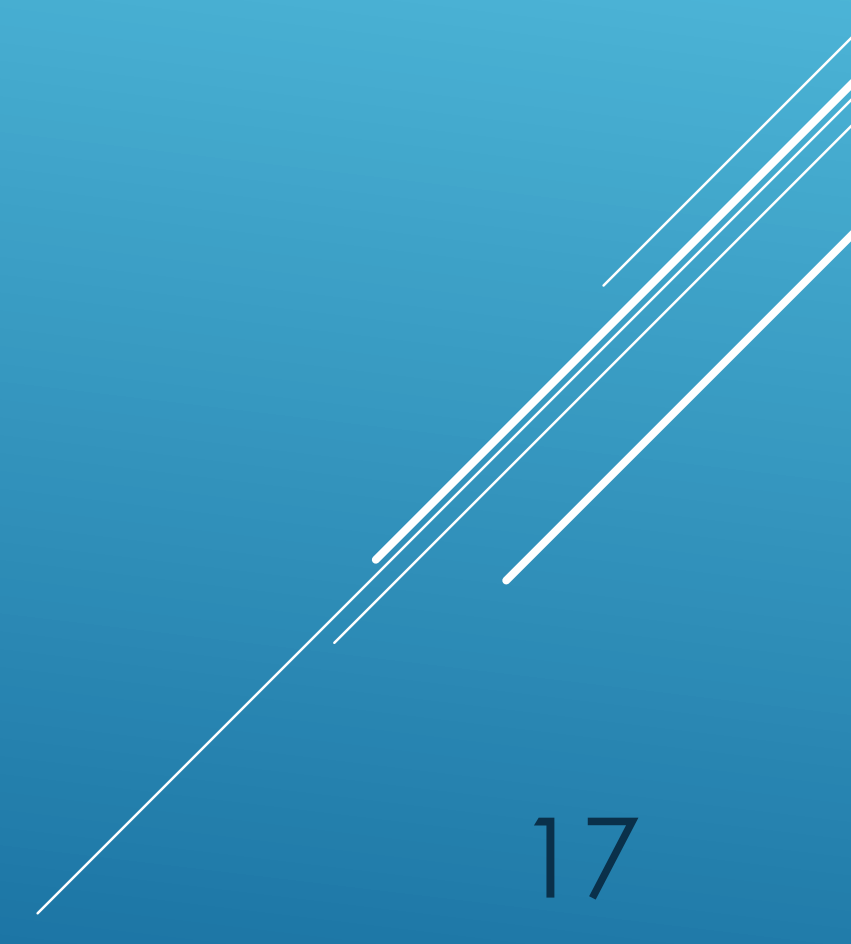


Numeric values (numbers having decimals) in $\mathrm{R}$ can pose a problem, as they are stored in the computer as fractions. This is called floating-point arithmetic. Roughly put, this means that the number has an imprecision of a scale of $10^{-15}$ of the unit (e.g. for a value of $1.00 \times 10^{25}$, the imprecision is of a scale of $10^{10}$ ). This is generally a minor problem for general calculations. However this can be challenging when trying to identify specific values, e.g. 0.1 will be understood in the computer in another way, which should look like this: 0.100000000000000561 . One way to avoid this problem (see the code in the next slide) is to round the values to an acceptable level, and to compare them using the all.equal() function, which checks for equality within numerical tolerance (at the opposite of the == comparison relational operator and of the identical() function). This offers a double security to insure that the possible accumulation of computational error will not affect the identification of equal values (see the code in the next slide).

\section{AVOIDING COMMON PROBLEMS: NUMERIC VALUES}




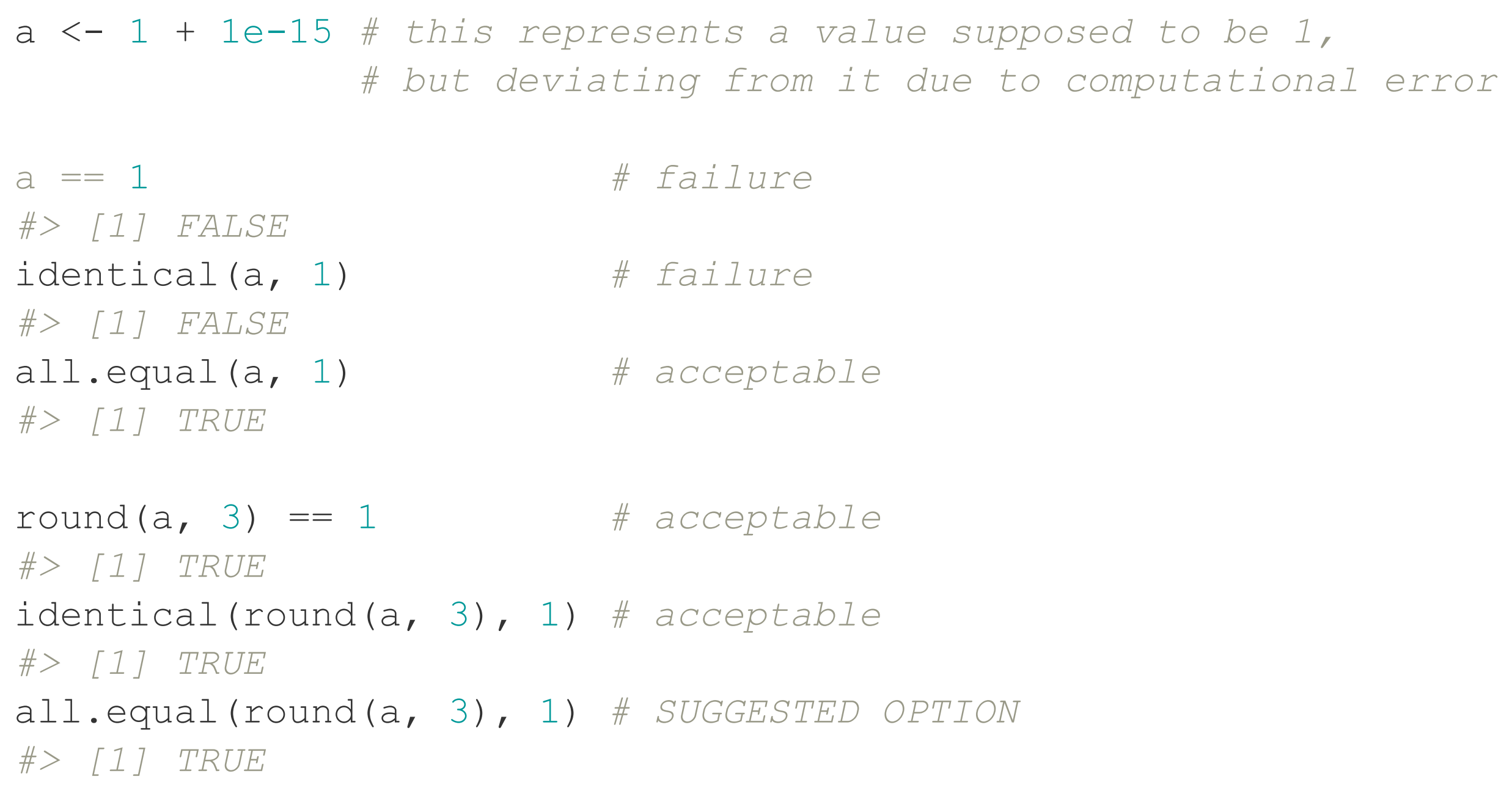

\section{AVOIDING COMMON PROBLEMS: NUMERIC VALUES}


$>$ How do I read text files?

Use the readlines() function for pure text, read.table(), read.csv(), read.fwf() to open respectively; tables where columns are separated by specific characters, csv files, and fixed-width columns tables.

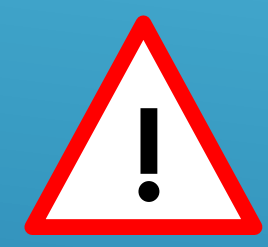

The stringsAsFactors parameter:

This parameter is present in most functions reading tables and creating data frames (i.e. the format for tables in R). By default it is generally set to TRUE. This means that each string (i.e. each element made up of text characters) will be set in another format, as a factor. This can be problematic: factors are not reacting similarly than characters in most functions. To avoid any problem it is here advised to set stringsAsFactors as FALSE when creating any data frame to avoid such problems.

\section{AVOIDING COMMON PROBLEMS: TEXT AND CHARACTERS}


- How do I identify specific characters sequences, for instance in a text file where the information is under the form 'data.1: 147'

Use pattern match and replacement, using for instance the grep(), grepl() and sub() functions, or the stringr package. Character pattern matching use characters having a specific meaning (such as $\wedge,[],,-,+$ and $\$$ in the example below). A sequence of characters used to identify a text pattern is called a regular expression. For more information on regular expression in $\mathbf{R}$ follow this link:

http://biostat.mc.vanderbilt.edu/wiki/pub/Main/SvetlanaEdenRFiles/regExprTalk.pdf

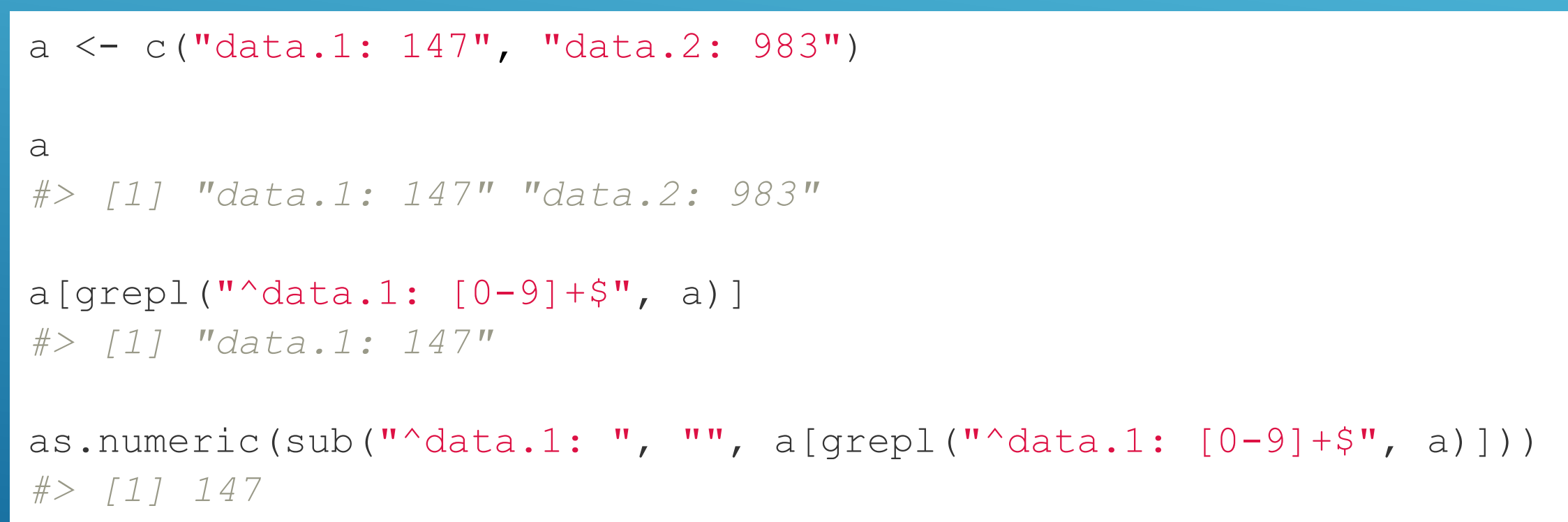

\section{AVOIDING COMMON PROBLEMS:} TEXT AND CHARACTERS 


\section{StratigrapheR}

A package to make lithologs

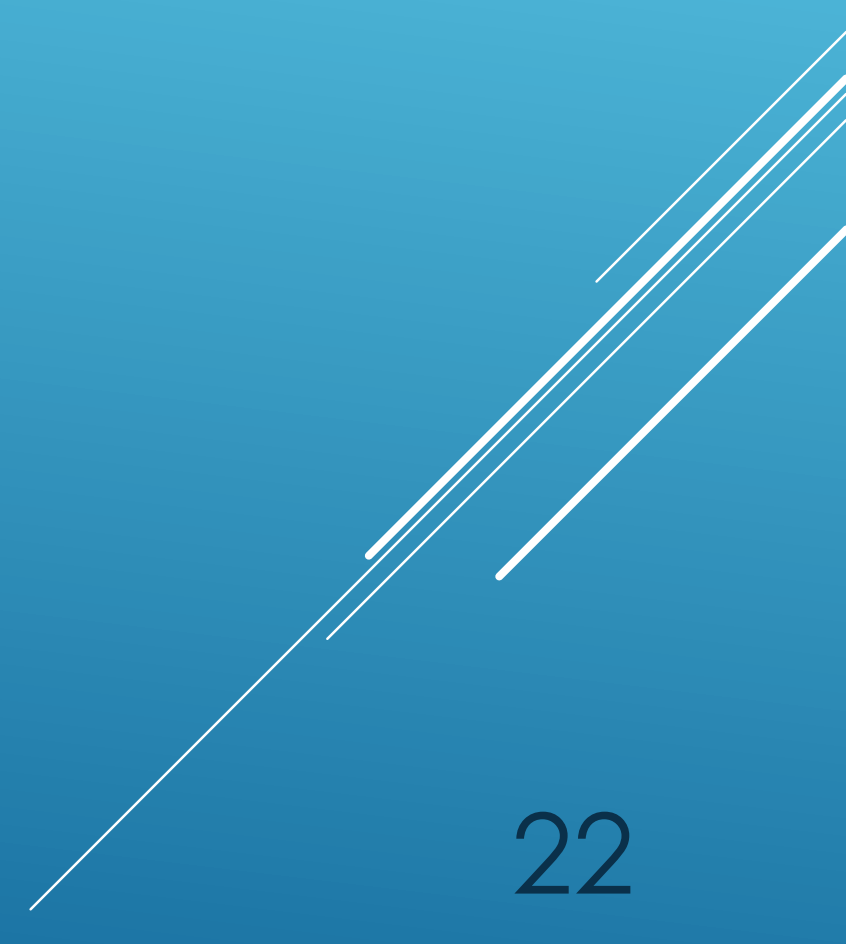




\section{WHY StratigrapheR ?}

- It allows to automate repetilitive drawings of lithologs

> It generates a basis for lithologs that can be improved in vector drawing software

- It allows to integrate lithologs to other data processing in $R$

- It can be made to evolve by anyone willing to learn $R$ 
- Working with stratigraphic data: the 'lim' object

- The lim object stores information of intervals: lower and upper boundaries ('l' and ' $r$ ', for left and right boundary), a rule for the inclusion of the boundaries ('b'), and an id ('id')
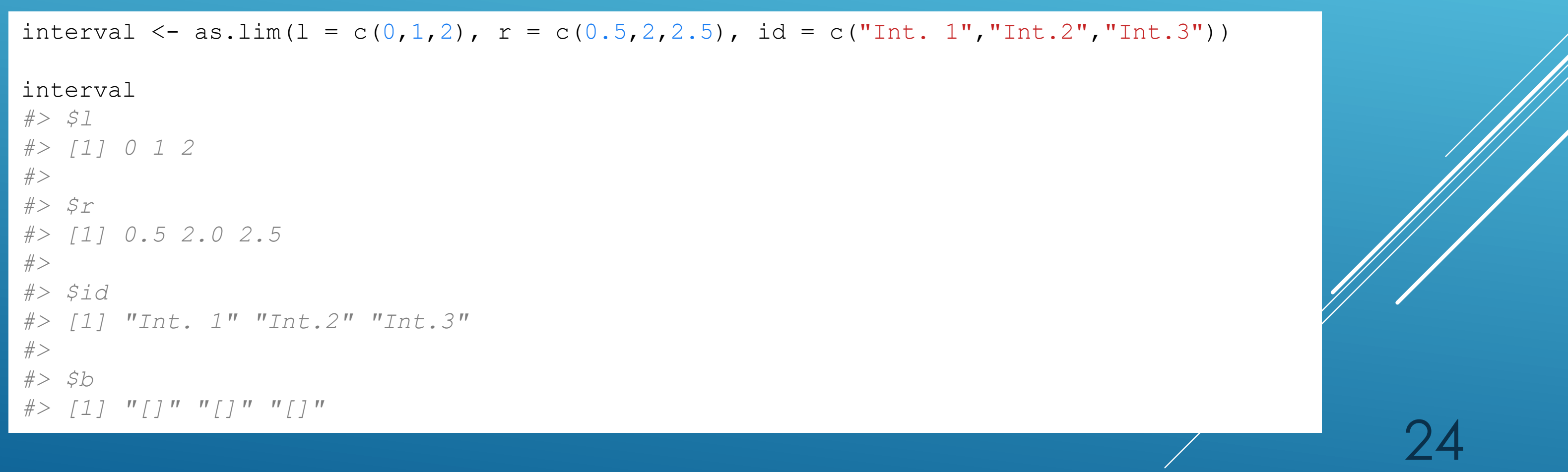
- The lim objects can be used for visualising intervals

interval $<-$ as.lim( $(=c(0,1,2), r=c(0.5,2,2.5), i d=c(" I n t .1 ", " I n t .2 ", " I n t .3 "))$

plot.new ()

plot.window (ylim $=c(-0.5,2.5), x l i m=c(0,2.5))$

axis $(3$, pos $=1.5$, las $=1)$

infobar $(\operatorname{ymin}=0, \operatorname{ymax}=1, x \operatorname{xin}=$ interval\$l, xmax $=$ interval\$r,

labels $=c($ interval\$id $)$, srt $=0)$

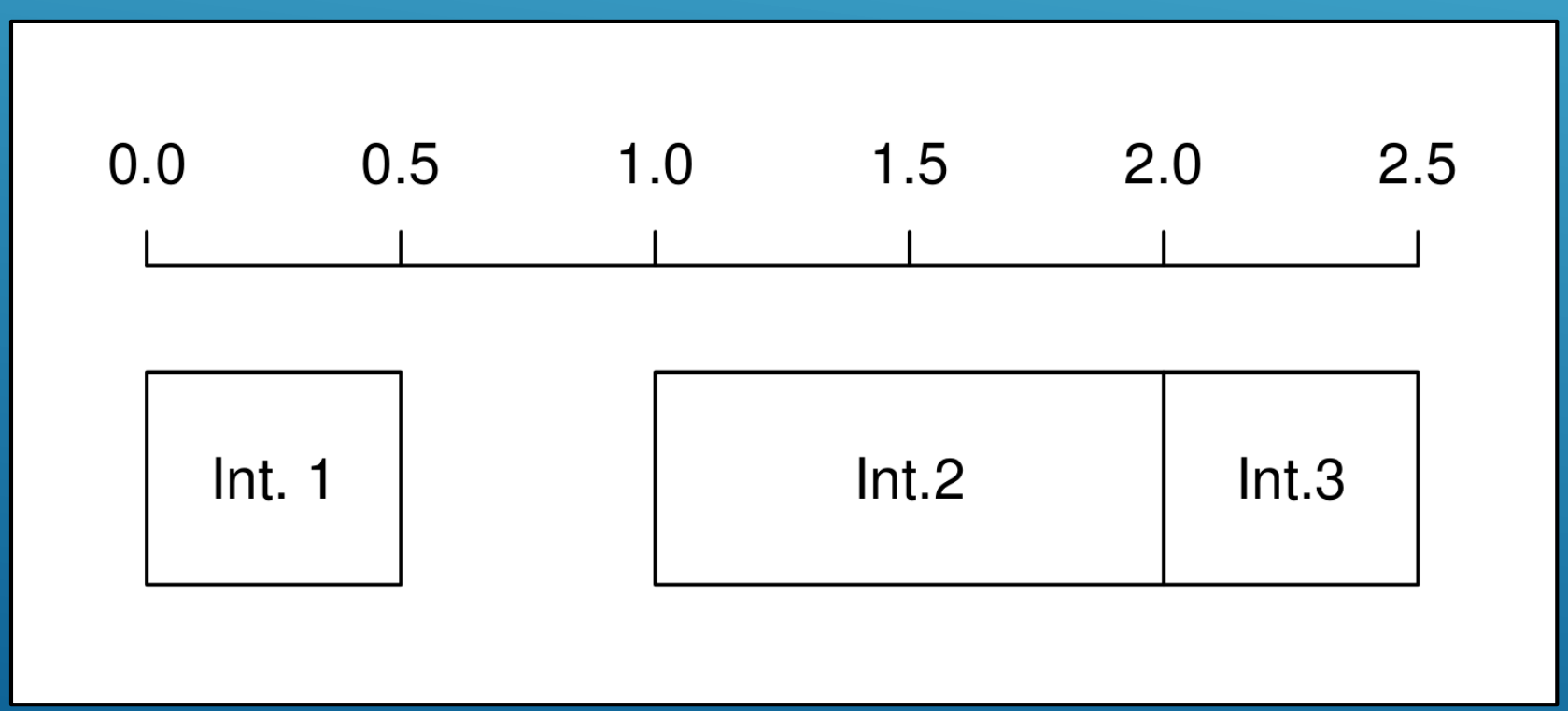


> The formalisation of intervals with the lim objects allows to define several functions: are.lim.nonunique() checks whether intervals are duplicated are.lim.nonadjacent() checks if the intervals share adjacent boundaries are.lim.distinct() checks whether the intervals are overlapping simp.lim() merges overlapping intervals having identical id flip.lim() finds the complementary intervals of a set of intervals (i.e. the gaps) mid.lim() defines intervals in between data points in.lim() finds which values belong to which intervals 
- The pdfDisplay() function can be used to generate and open plots of any size

- The plot.new() and plot.window() functions are used to introduce an empty plot

- The minorAxis() function allows to have an axis with minor ticks

graphical_function $<-$ function()

\{

opar <- par() \$mar \# Save initial graphical parameters

$\operatorname{par}(\operatorname{mar}=\mathrm{c}(2,3,0,1))$

plot.new ()

plot.window $(x \lim =\mathrm{c}(0,1), \mathrm{ylim}=\mathrm{c}(0,1))$

axis (1)

minorAxis $(2$, at.maj $=\operatorname{seq}(0,1,0.5), \mathrm{n}=5$, las $=1)$ points $(\mathrm{c}(0.25,0.75), \mathrm{c}(0.75,0.75), \mathrm{pch}=19)$

polygon (c(0.1, $0.25,0.75,0.9,0.75,0.25)$, $c(0.5,0.25,0.25,0.5,0.4,0.4), \quad 1 w d=2)$

par (mar = opar) \# Restore initial graphical parameters

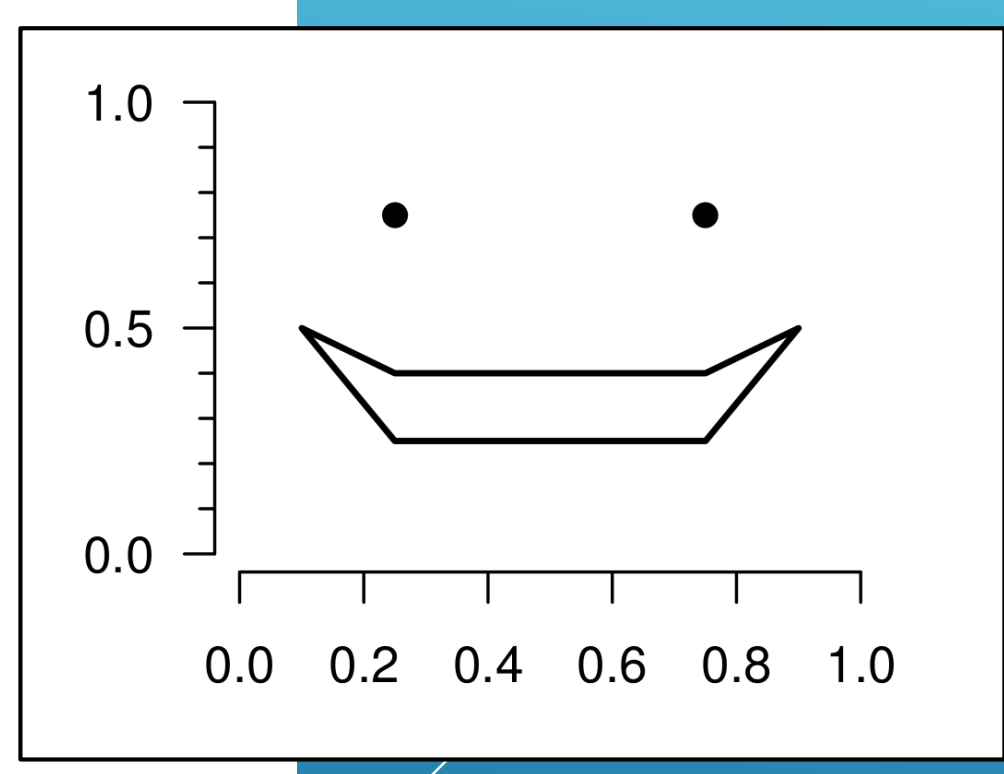
\}

pdfDisplay (graphical_function(),"graphical_function", width = 3 , height $=\overline{2}$ ) 
- The multigons() function plots several polygons at once

i $<-c(\operatorname{rep}(" \mathrm{~A} 1 ", 6), \operatorname{rep}(" \mathrm{~A} 2 ", 6), \operatorname{rep}(" \mathrm{~A} 3 ", 6))$ \# Polygon ids $\mathrm{x}<-\mathrm{c}(1,2,3,3,2,1,2,3,4,4,3,2,3,4,5,5,4,3)$ \# $x$ coordinates $\mathrm{y}<-\mathrm{c}(1,2,3,4,5,6,1,2,3,4,5,6,1,2,3,4,5,6)$ \# y coordinates

plot.new ()

plot.window $(x \lim =c(0,6), y \operatorname{yim}=c(0,7))$ multigons (i, $x, y$,

front = "A2", \# This gets the polygon A2 in front of all others

density $=\mathrm{C}(\mathrm{NA}, 5,10)$, \# Different shading density

scol = "grey8o", \# Same shading color

col = c("black", "grey2o", "white"), \# Different background color

lwd $=2$, \# width of border lines for all polygons

slty $=2$, \# shading lines type, same for all polygons

slwd $=1$ ) \# Shading lines width, same for all polygons

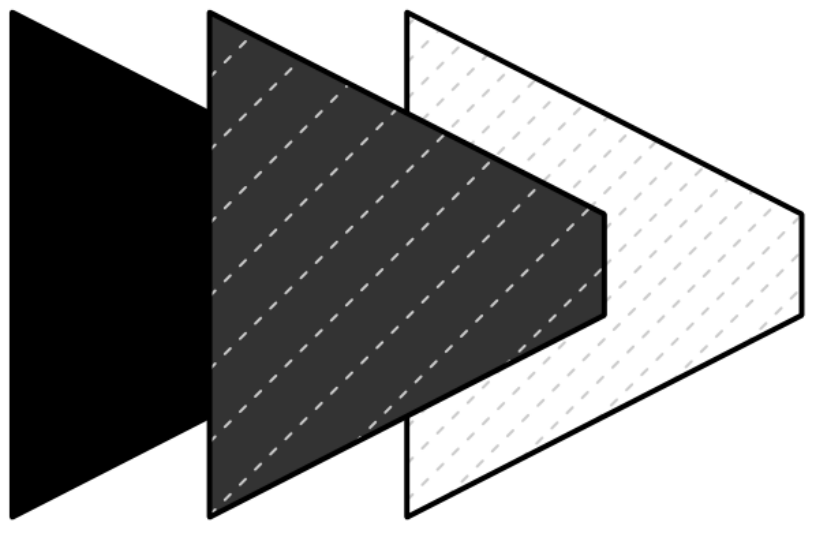

28 
> The multilines() function plots several polylines at once

$$
\begin{aligned}
& i<-c(r e p(" A 1 ", 6), \quad r e p(" A 2 ", 6), \quad r e p(" A 3 ", 6)) \\
& x<-c(1,2,3,3,2,1,4,5,6,6,5,4,7,8,9,9,8,7) \\
& \text { y<-c }(1,2,3,4,5,6,1,2,3,4,5,6,1,2,3,4,5,6)
\end{aligned}
$$

\section{plot.new ()}

plot.window $(x \lim =\mathrm{c}(0,10), \mathrm{ylim}=\mathrm{c}(0,7))$

multilines(i, $x, y, j=c(" A 3 ", " A 1 ", " A 2 "), l t y=c(1,2,3), l w d=2$,

$$
\text { type }=c(" 1 ", " \circ ", " \circ "), \text { pch }=c(N A, 21,24), \text { cex }=1, \text { bg }=\text { "black") }
$$

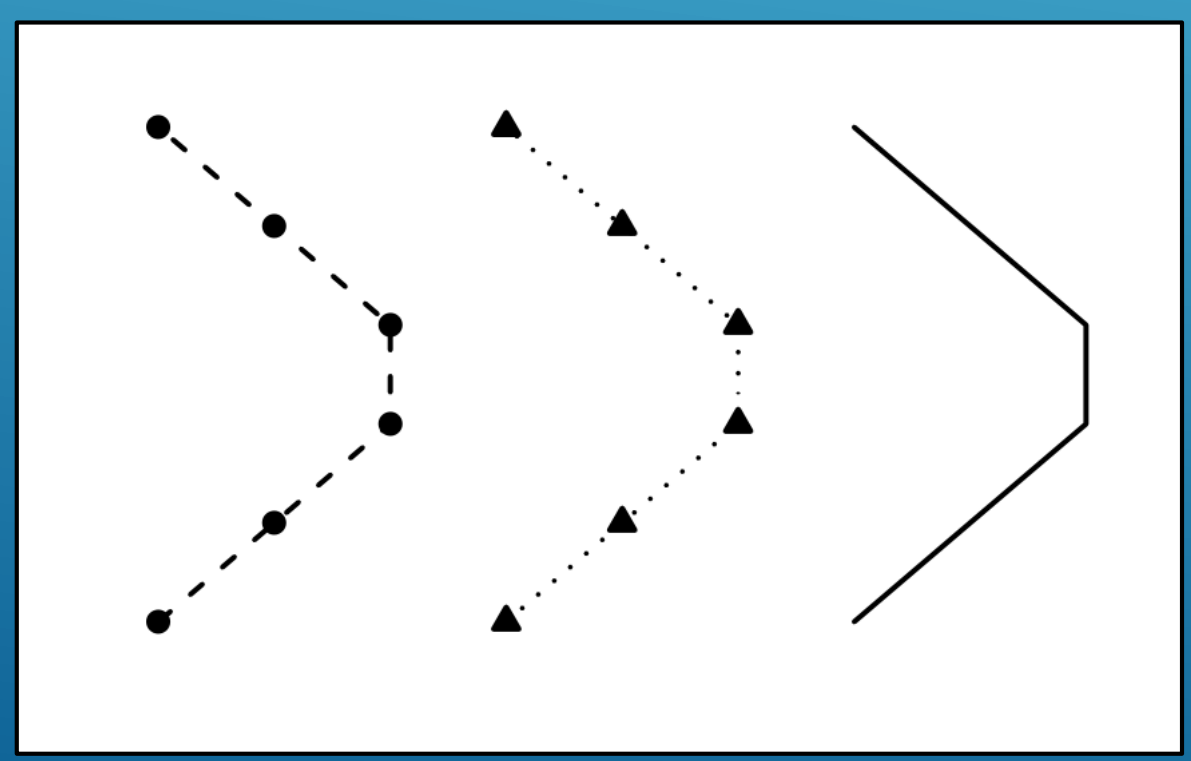


- Pre-drawn SVG objects can be imported using the pointsvg() function

> These SVG objects can be directly drawn using centresvg() or framesvg()

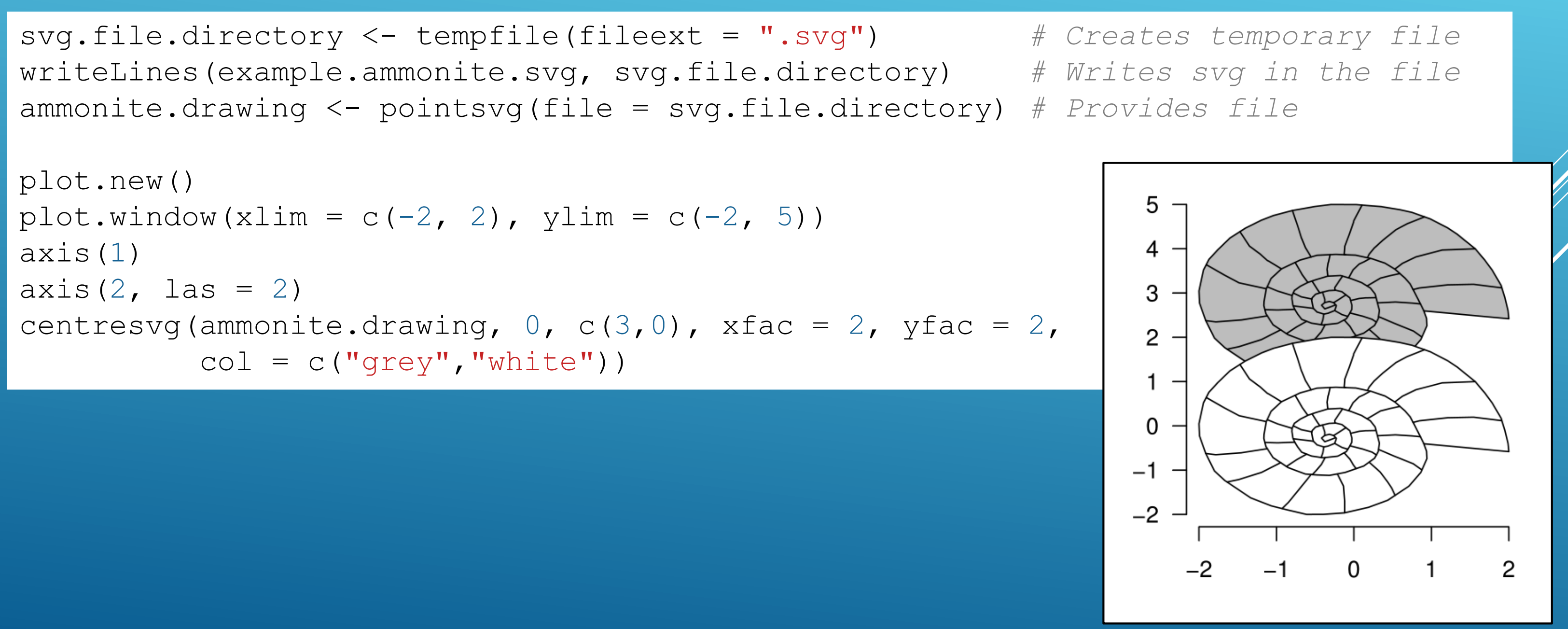


> Using centresvg() or framesvg() allows to plot the SVG object several times with one call of the function

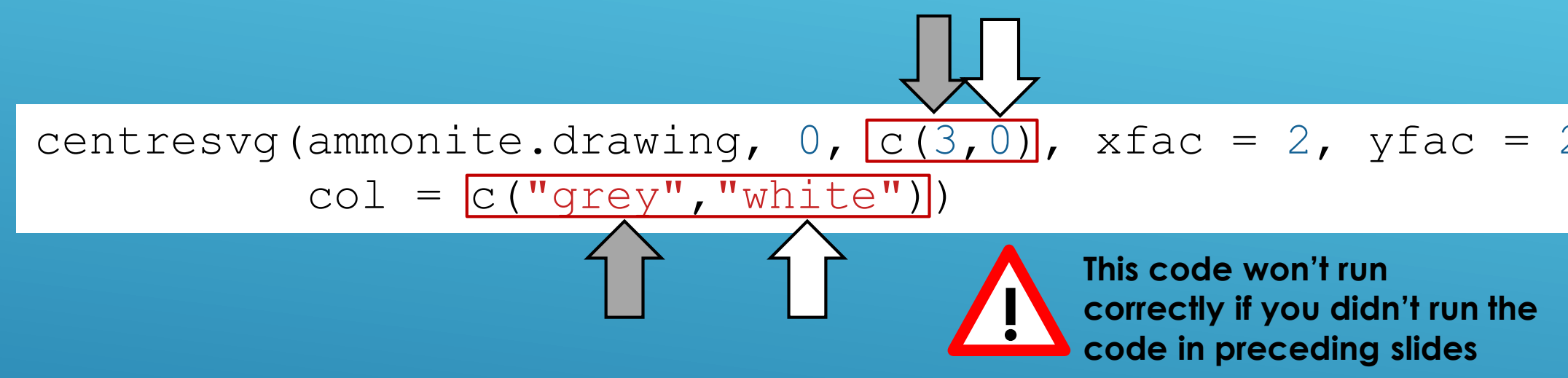

- The changesvg() function can change the SVG object:

- Change of the order of plotting of the polylines and polygons

- Removal of some of the polylines or polygons

- Inversion of the figure in $x$ and/or $y$

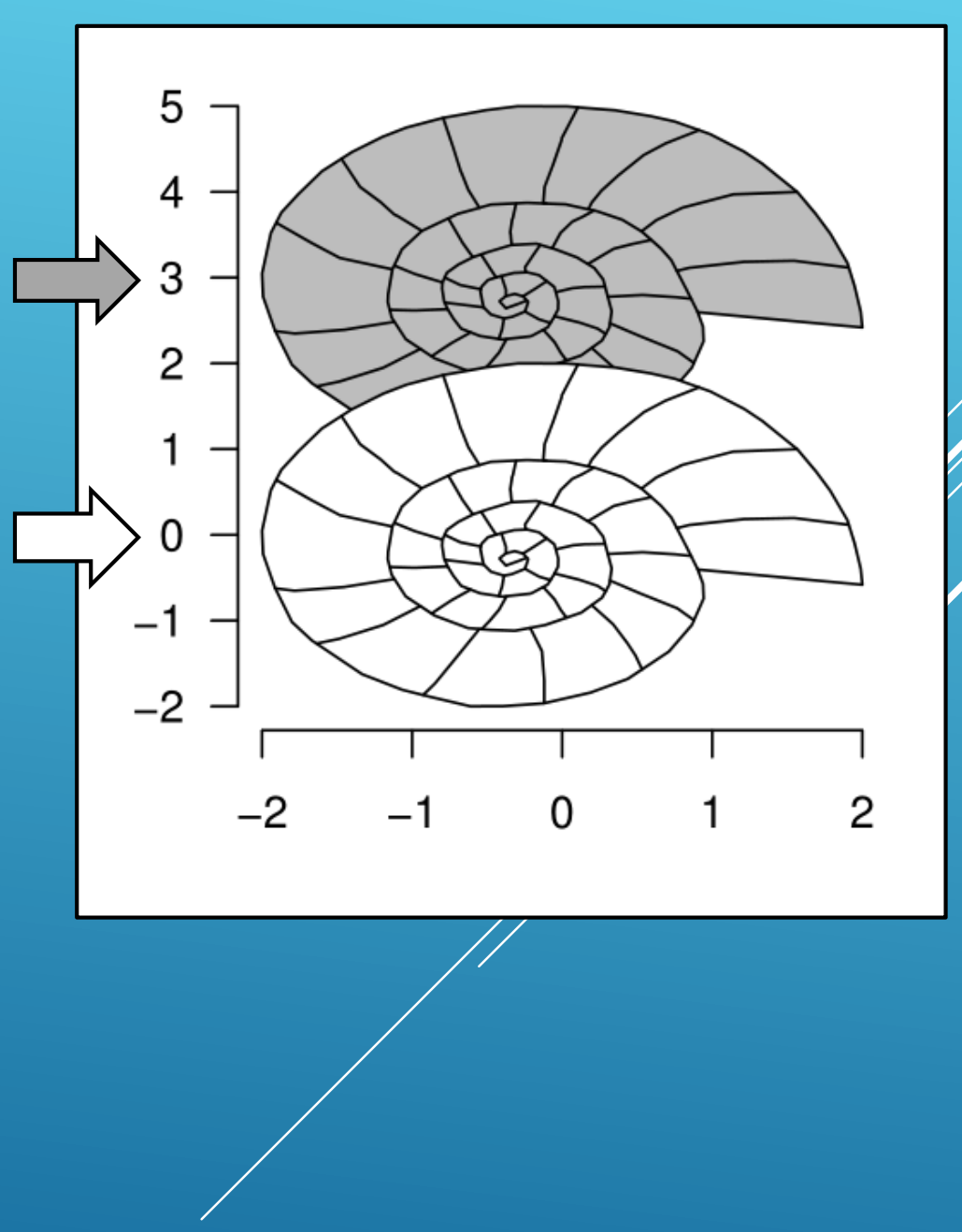


- The information for the beds in a litholog can be provided as a table (which in $\mathrm{R}$ corresponds to a data frame object):

\begin{tabular}{|c|c|c|c|c|c|}
\hline id & I & r & h & colour & litho \\
\hline B1 & 0 & 1 & 3 & grey & S \\
\hline B2 & 1 & 3 & 4 & grey & L \\
\hline B3 & 3 & 4 & 5 & black & C \\
\hline B4 & 4 & 9 & 4 & white & L \\
\hline B5 & 9 & 11 & 4 & white & L \\
\hline
\end{tabular}

information for each bed: id identifies each bed, I and r provide the lower and upper boundaries, $\mathrm{h}$ the hardness, and the colour is provided along with a code for lithology (S for shale, L for Limestone, C for chert).

- Such a table is provided in StratigrapheR as an example:

View (bed. example) 
- The data frame describing the beds can be merged with a legend data frame to attribute a symbology to the beds, based on a common column (in our case, litho, standing for lithology)

\begin{tabular}{|c|c|c|c|c|c|}
\hline \multirow{2}{*}{ View (legend) } & \multicolumn{5}{|c|}{$\begin{array}{l}\text { (litho = c("S", "L", "C" } \\
\text { col = c ("grey30", "grey } \\
\text { density }=c(30,0,10), \\
\text { angle }=c(180,0,45),\end{array}$} \\
\hline & $\Delta$ & litho & col $=$ & density $=$ & angle \\
\hline & 1 & s & greyз0 & 30 & 180 \\
\hline & 2 & L & grey90 & 0 & 0 \\
\hline & 3 & c & white & 10 & 45 \\
\hline
\end{tabular}

- The merging is done here by the left_join() function of the dplyr package

\section{library (dplyr)}

bed.legend <- left_join(bed.example, legend, by = "litho")

View (bed.legend)

\begin{tabular}{|c|c|c|c|c|c|c|c|c|c|}
\hline 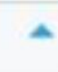 & id $=$ & $1=$ & $r=$ & $\mathbf{h}=$ & colour $=$ & litho $=$ & col $=$ & density $=$ & angle \\
\hline 1 & $\mathrm{~B} 1$ & 0 & 1 & 3 & grey & $\mathrm{s}$ & grey30 & 30 & 180 \\
\hline 2 & B2 & 1 & 3 & 4 & grey & L & grey90 & 0 & 0 \\
\hline 3 & B3 & 3 & 4 & 5 & black & C & white & 10 & 45 \\
\hline 4 & B4 & 4 & 9 & 4 & white & L & grey90 & 0 & 0 \\
\hline 5 & B5 & 9 & 11 & 4 & white & L & grey90 & 0 & 0 \\
\hline
\end{tabular}


- Based on the information of the provided data frames, polygons coordinates can be defined using the litholog() function, and its output can be plotted with the correct symbology using multigons(). Text can be added in the beds using bedtext()

basic.log <- litholog $(1=$ bed.example\$l, $r=$ bed.example\$r,

$\mathrm{h}=\mathrm{bed}$.example\$h, $\mathrm{i}=$ bed.example\$id)

plot.new ()

plot.window $(x \lim =\mathrm{c}(0,6), \mathrm{ylim}=\mathrm{c}(-1,77))$

minorAxis (2, at.maj $=\operatorname{seq}(0,75,5), \mathrm{n}=5)$

multigons(basic.log\$, $x=$ basic.log $\$ x y, y=b a s i c \cdot \log \$ d t$, $\mathrm{col}=$ bed.legend\$Col,

density = bed.legend\$density,

angle $=$ bed.legend\$angle)

bedtext (labels = bed.example\$id,

$l=$ bed.example\$l,

$r=$ bed.example $\$ r$,

$\mathrm{x}=0.5$, \# $\mathrm{x}$ position where to centre the text

ymin = 3) \# ymin defines the minimum thickness

\# for the beds where text can be added

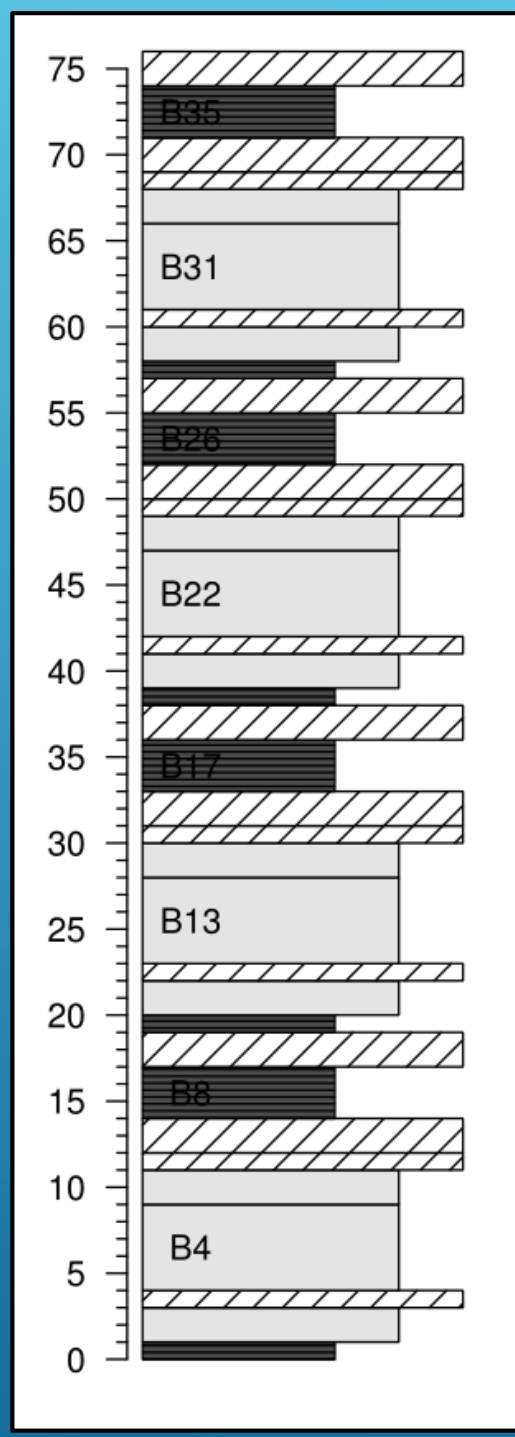

This code won't run

correctly if you didn't run the code in preceding slides 
> A litholog created via the litholog() function can be modified. To add thickness variations to beds, the weldlog() function can be used:

s1 $<-\operatorname{sinpoint}(5,0,0.5$, nwave $=1.5)$

s2 <- sinpoint $(5,0,1$, nwave $=3$, phase $=0$ )

s3 <- framesvg (example.liquefaction, 1, 4, 0, 2, plot = FALSE, output = TRUE)

final.log $<-$ weldlog(log = basic.log, dt = boundary.example\$dt,

$$
\begin{aligned}
& \text { seg }=\text { list }(\mathrm{s} 1=\mathrm{s} 1, \mathrm{~s} 2=\mathrm{s} 2, \mathrm{~s} 3=\mathrm{s} 3) \text {, } \\
& j=c(" s 1 ", " s 1 ", " s 1 ", " s 3 ", " s 2 ", " s 2 ", " s 1 "), \text { warn = FALSE) }
\end{aligned}
$$

plot.new ()

plot.window $(x \lim =c(0,6), y \lim =c(-1,77))$

minorAxis (2, at.maj $=\operatorname{seq}(0,75,5), \mathrm{n}=5$, las $=1$ )

multigons(final.log\$i, $x=f i n a l . \log \$ x y, y=f i n a l . l o g \$ d t$,

col $=$ bed.legend\$col,

density = bed.legend\$density,

angle $=$ bed.legend\$angle)

bedtext ( $l$ abels $=$ bed.example\$id, $l=$ bed.example\$l, $r=$ bed.example $\mathbf{r}$,

$$
\mathrm{x}=0.75, \operatorname{ymin}=3 \text { ) }
$$

\section{This code won't run
correctly if you didn't run the

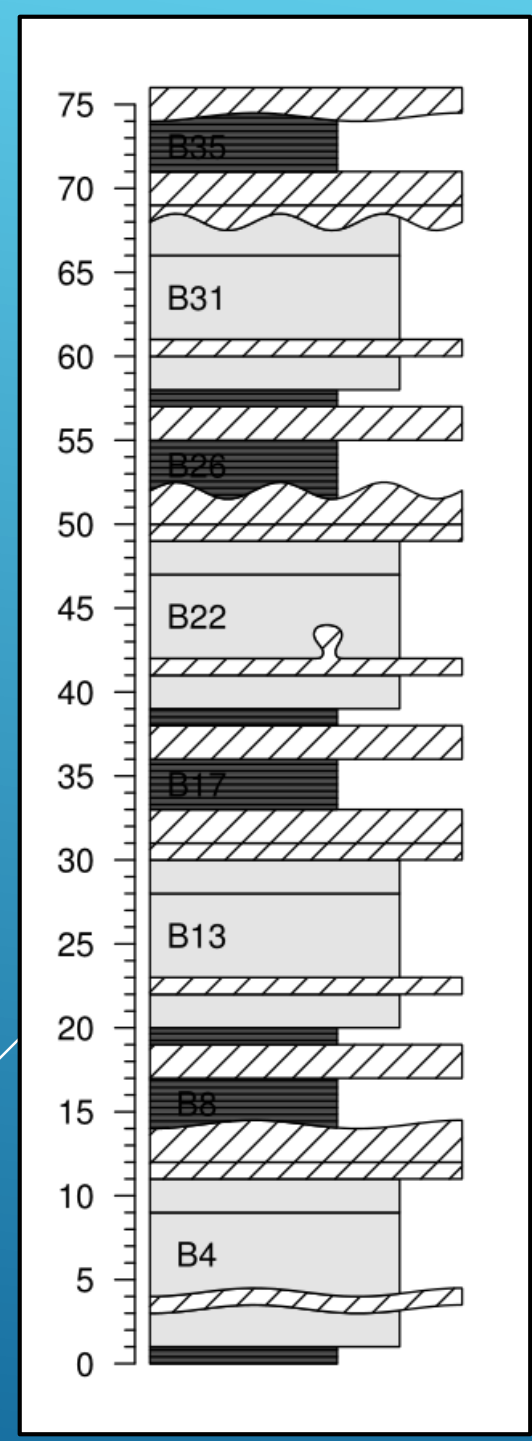


The litholog plotting code can be set into a function, and used to plot only a part of the log, to be able to latter plot it onto several pages (Using for instance LaTeX)

$\log$. function $<-$ function $(x \lim =c(-2.5,7), y \lim =c(-1,77))$

i

plot.new ()

plot.window $(x \lim =x \lim , y \lim =y \lim )$

minorAxis $(2$, at.maj $=\operatorname{seq}(0,75,5), \mathrm{n}=5$, pos $=-1.75$, las $=1)$

multigons(final.log\$i, $x=$ final.log $\$ x y, y=f i n a l \cdot \log \$ d t$

$\operatorname{col}=$ bed.legend\$col,

density = bed.legend\$density

angle $=$ bed.legend\$angle)

bedtext (labels = bed.example\$id, $l=$ bed.example $\mathbf{l}, r=$ bed.example $\mathbf{r}$,

edge $=2$,

centresvg (example.ammonite, 6

fossil.example\$dt[fossil.example\$type == "ammonite"], xfac $=0.5$ )

centresvg (example.belemnite, 6,

fossil.example\$dt[fossil.example\$type == "belemnite"], $\mathrm{xfac}=0.5)$

infobar $(-1.5,-1$, chron.legend\$l, chron.legend\$r,

labels = chron. legend\$id,

$m=$ list $(\mathrm{col}=$ chron. legend $\$ \mathrm{bg} \cdot \mathrm{col})$,

infobar $(-0.25,-0.75$, bed.example $\$ 1$, bed.example $\$ r$

\} $\mathrm{m}=$ list $(\mathrm{col}=\operatorname{colour}))$

gr $<-$ function ()

par <- par() \# Save initial graphical parameters

$\operatorname{par}(\operatorname{mar}=c(1,2,1,2)$, yaxs $=" i ")$

for (i in $1: 0)$

ylim $<-c(0,40)$

$\log$. function $(y \lim =y l i m+40 * i)$

par (mar = opar\$mar, myaxs = opar\$yaxs) \# Restore initial graphical parameters \}

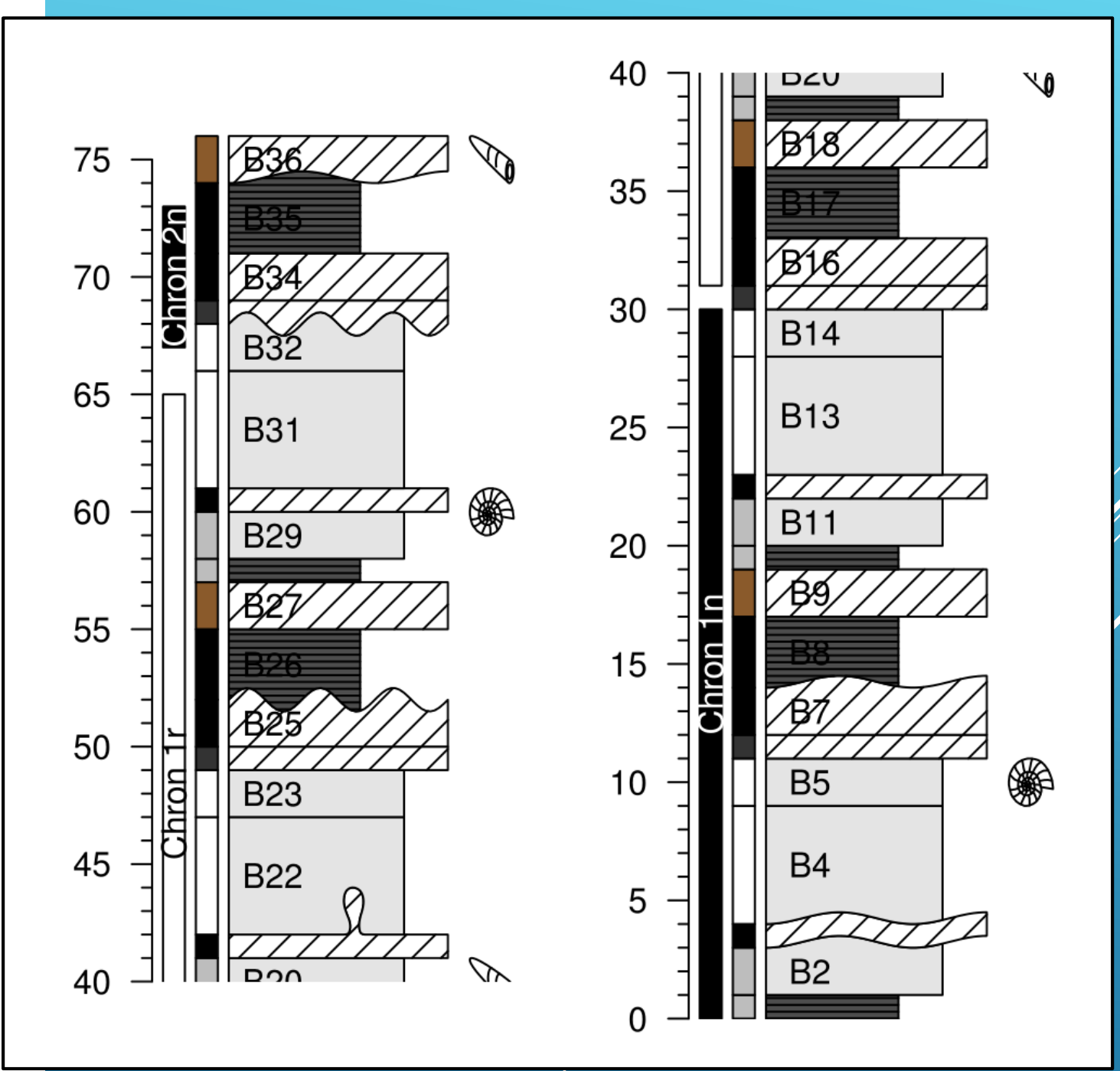

This code won't run
correctly if you didn't run the code in preceding slides 
Any other plot(s) can be added along the litholog, using for instance the par() function. Great care should be taken to insure that the depth axis is similar

\# Code repeated from earlier examples ----

basic.log <- litholog( $1=$ bed.example\$l, $r=$ bed.example\$r,

$h=$ bed.example\$h, $i=$ bed.example\$id)

legend <- data.frame (litho $=\mathrm{C}(\mathrm{S} ", \mathrm{~L} ", \mathrm{C} ")$

col = c("grey3o", "grey90", "white")

density $=c(30,0,10)$

angle $=c(180,0,45)$, stringsAsFactors = FALSE $)$

bed.legend <- dplyr: :left join(bed.example, legend, by = "litho")

s1 $<-$ sinpoint $(5,0,0.5$, nwave $=1.5)$

s2 $<-$ sinpoint $(5,0,1$, nwave $=3$, phase $=0)$

s3 <- framesvg (example.liquefaction, 1, 4, 0, 2, plot = FALSE, output = TRUE)

final.log $<-$ weldlog(log = basic.log, $d t=$ boundary.example $\$ d t$

seg $=$ list $(\mathrm{s} 1=\mathrm{s} 1, \mathrm{~s} 2=\mathrm{s} 2, \mathrm{~s} 3=\mathrm{s} 3)$,

$j=c(" s 1 ", " s 1 ", " s 1 ", " s 3 ", " s 2 ", " s 2 ", " s 1 ")$, warn = F)

opar <- par() \$mfrow \# Save initial graphical parameters

par (mfrow $=c(1,2)$ )

plot.new ()

plot.window $(x \lim =c(0,6), y \lim =c(-1,77))$

minorAxis (2, at.maj $=\operatorname{seg}(0,75,5), \mathrm{n}=5$, las $=1$ )

multigons(final.log\$i, $x=$ final.log\$xy, $y=f i n a l . l o g \$ d t$

$\operatorname{col}=$ bed.legend $\$ \mathrm{COl}$

density = bed.legendsdensity,

angle $=$ bed.legend\$angle)

bedtext (labels = bed.example\$id, $l=$ bed.example\$l, $r=$ bed.example\$r,

$$
=0.75, \operatorname{ymin}=3 \text { ) }
$$

plot.new ()

plot. window $\left(x \lim =c\left(-2 * 10^{\wedge}-8,8 * 10^{\wedge}-8\right), y \lim =c(-1,77)\right)$

minorAxis $(4$, at.maj $=\operatorname{seq}(0,75,5), \mathrm{n}=5$, las $=1)$

lines (proxy.example\$ms, proxy.example\$dt, type = "०", pch = 19)

$\operatorname{axis}(1)$

title (xlab = "Magnetic Susceptibility")

par (mfrow = opar) \# Restore initial graphical parameters
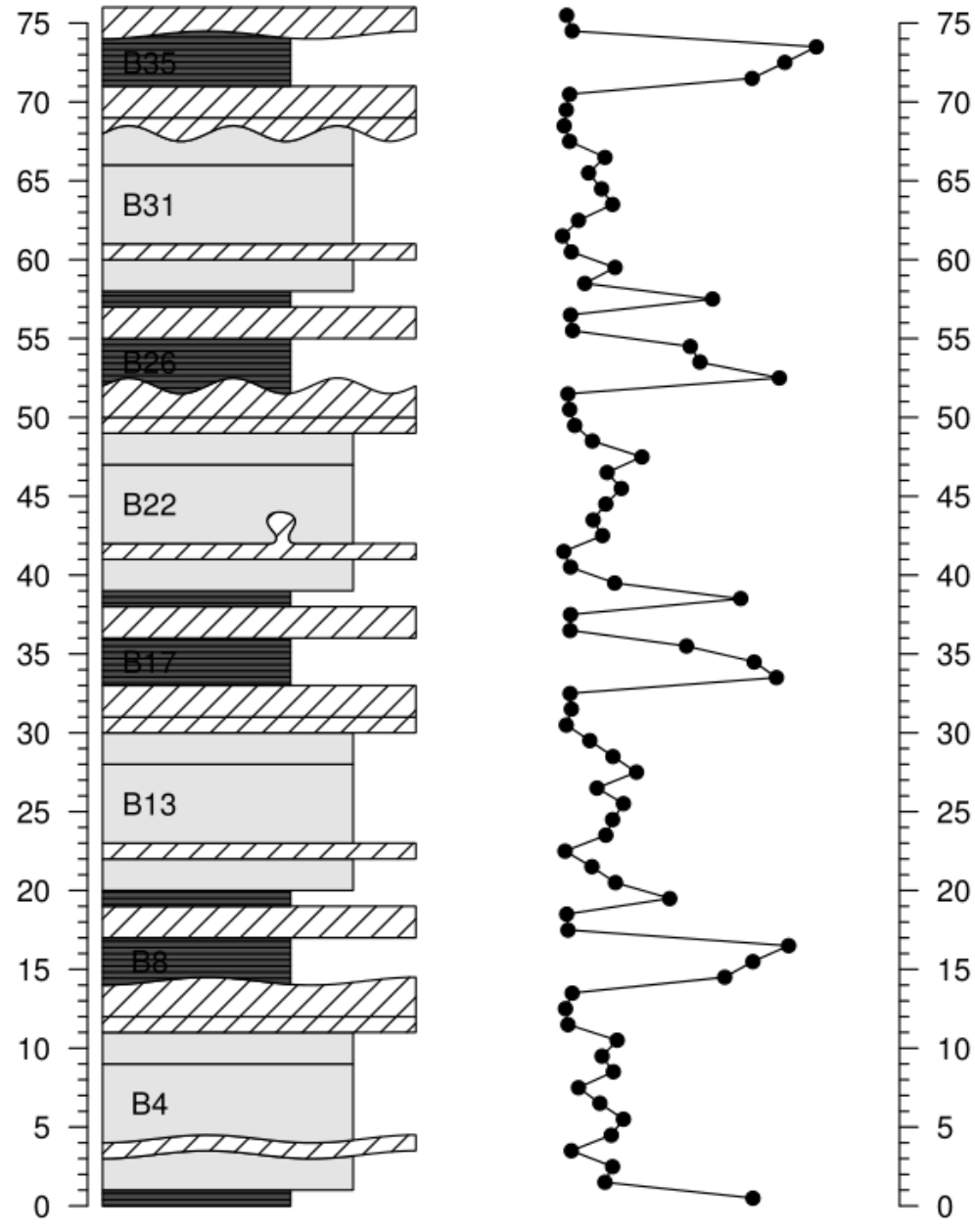

$08-08-08$

Magnetic Susceptibility 


\section{A legend can be created using nlegend(). Each symbol is in another plot, and is centred} on the coordinates $(0,0)$.

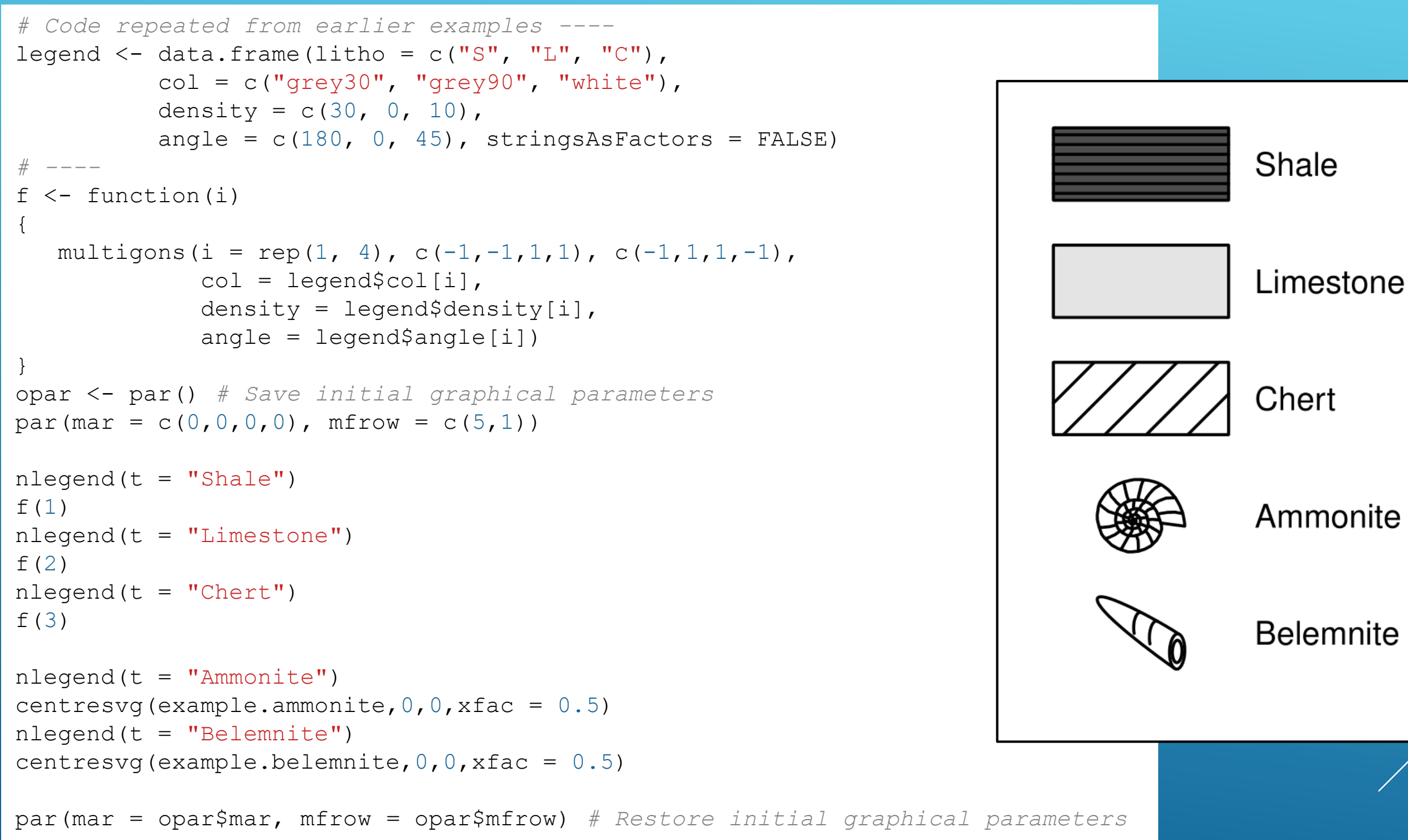

Belemnite 
- Another resource is available for StratigrapheR:

https://orbi.uliege.be/bitstream/2268/237418/1/Poster\%20STRATI\%202019\%20V3.pdf
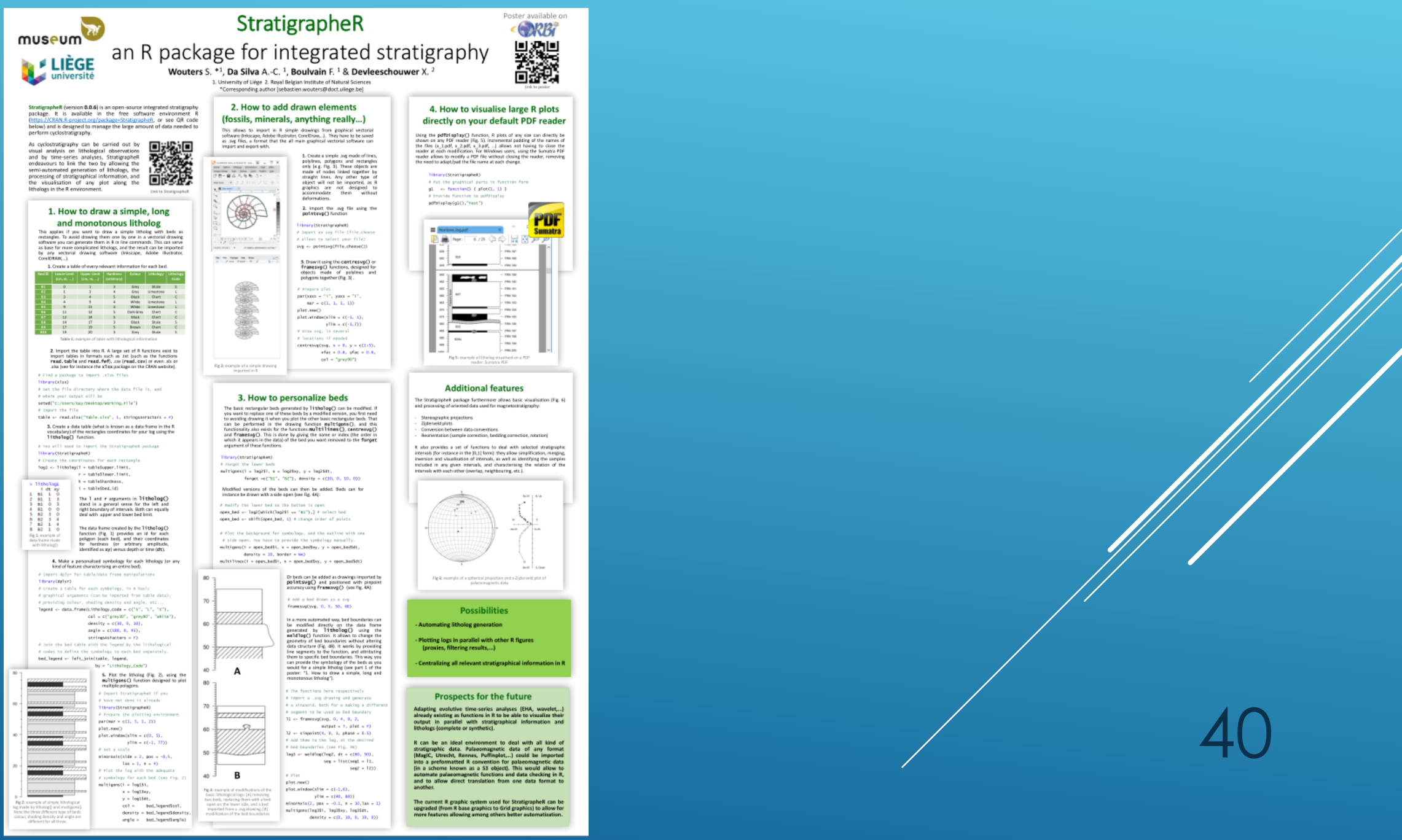


\section{PLEASE}

\section{HELP ME,}

MY CODE DOES NOT WORK

AND I AM DESPERATELY STUCK !แ!

Self-explanatory 


\section{PLEASE HELP ME, MY CODE DOES NOT WORK AND I AM DESPERATELY STUCK !!!!}

- Starting to code in any language is difficult, and everyone rising to the challenge will sooner or later be faced with what seems to be an insurmountable task. On a personal level I can say without a doubt that I wouldn't have been able to code what I wanted to code without the help of one of my relatives, who is a trained programmer that I can annoy with my $\mathbf{R}$ problems every now and then. Seeking help is actually a full part of the job of programmer. Thankfully there are a few resources available out there:

- Google (or any other search engine, it is basic but efficient)

- Stack Overilow https://stackoverflow.com/questions/tagged/r

- The maintainer of the package you are using

- To ask questions, is it best to provide a reprex, which stands for "representative example": https://community.rstudio.com/t/faq-whats-areproducible-example-reprex-and-how-do-i-do-one/5219

- If you have any question on StratigrapheR, please contact the maintainer: sebastien.wouters@doct.uliege.be

- You can post your question on Stack Overflow, and link the question to the maintainer, so that other users can take advantage of the answer

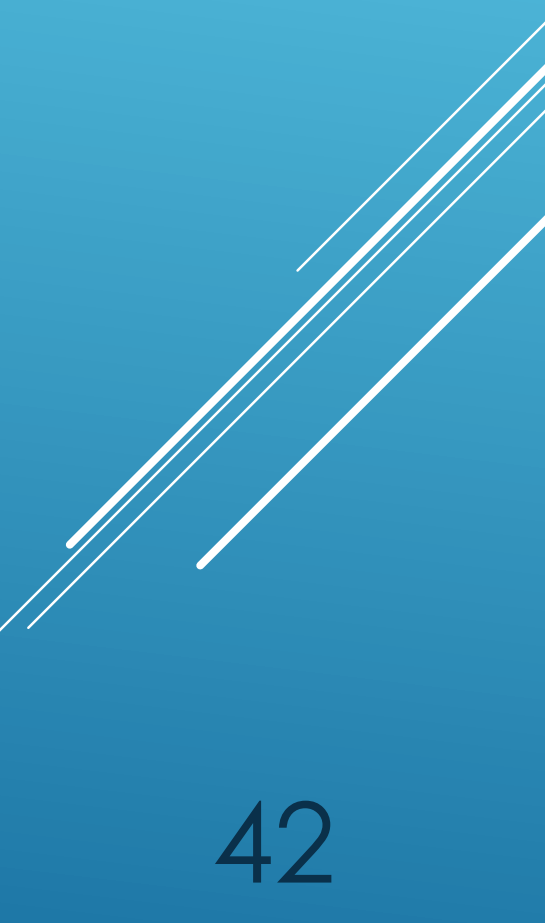

